\title{
An unusual distribution of the niacin receptor in cattle
}

\author{
E. C. Titgemeyer, ${ }^{1}$ L. K. Mamedova, K. S. Spivey, J. K. Farney, and B. J. Bradford \\ Department of Animal Sciences and Industry, Kansas State University, Manhattan 66506
}

\begin{abstract}
Responses to pharmacological doses of niacin, an agonist for GPR109A (niacin receptor), were different in cattle than in humans and rodents. Thus, the tissue distribution of GPR109A was investigated in cattle. Samples of tail head fat, back fat, perirenal fat, longissimus muscle, and liver were analyzed for abundance of GPR109A mRNA by quantitative real-time reverse transcription-PCR and for abundance of GPR109A protein by Western blotting. Niacin receptor transcript and protein were detected in all tissues analyzed. The mRNA for GPR109A was more abundant in liver than in the other tissues sampled (GPR109A:RPS9 mRNA abundance $=0.56$ in liver compared with 0.06 in longissimus muscle, 0.15 in kidney fat, 0.11 in back fat, 0.23 in tail head fat; standard error of the mean = 0.028). Additionally, mRNA for GPR109A was found (GPR109A:RPS9 mRNA abundance $\geq 0.004$ ) in each of the 5 regions of bovine brain that were analyzed: cerebral cortex, cerebellum, thalamus, hypothalamus, and brain stem. Evaluation of liver tissue by immunofluorescence suggested that GPR109A was expressed in parenchymal cells and not localized exclusively to immune-system cells. Finally, analysis of the putative bovine GPR109A sequence verified that AA residues required for binding niacin in human GPR109A are conserved, suggesting that the bovine sequence identified encodes a functional niacin receptor. The identification of GPR109A in bovine liver, muscle, and brain is a novel finding.
\end{abstract}

Key words: nicotinic acid, niacin receptor (GPR109A), bovine

\section{INTRODUCTION}

Lee et al. (2001) first identified GPR109A as a receptor for nicotinic acid (NA; niacin) in mice. Although GPR109A and GPR109B are similar, sharing 96\% AA sequence identity, GPR109B was identified as a low-

Received January 19, 2011.

Accepted June 1, 2011.

${ }^{1}$ Corresponding author: etitgeme@ksu.edu affinity receptor for NA (Wise et al., 2003). The NA receptor GPR109A is predominantly localized in adipose tissue and immune cells in humans (Wise et al., 2003) and mice (Tunaru et al., 2003), but has been found in a wide variety of tissues such as murine hepatocytes (Li et al., 2010), retinal pigment epithelium (Martin et al., 2009), intestinal epithelium in mice and humans (Thangaraju et al., 2009), and in human brain (Miller and Dulay, 2008). The endogenous ligand for GPR109A is $\beta$-hydroxybutyrate (Gille et al., 2008).

At pharmacological doses (i.e., those that would lead to effects mediated by stimulation of GPR109A), NA inhibits lipolysis in cattle (Pires and Grummer, 2007; Pires et al., 2007), presumably by acting on GPR109A, a Gi-coupled receptor (Gille et al., 2008). Activation of GPR109A by NA leads to inhibition of adenylyl cyclase activity and, subsequently, a decrease in cyclic AMP (cAMP) concentrations within the cell. Decreases in cAMP in adipocytes lead to sequelae (inactivation of protein kinase A and decreased phosphorylation of hormone sensitive lipase) that reduce lipolysis. Li et al. (2010) demonstrated that although the basal expression of GPR109A in murine liver was low, its activation with NA decreased cAMP concentrations in hepatocytes and affected cholesterol metabolism.

Some of the cattle from which samples were analyzed for the work described herein were initially used in research designed to determine if NA could reduce lipolysis induced by $\beta$-adrenergic agonists, which, upon stimulation of $\beta$-adrenergic receptors, lead to effects opposite of GPR109A stimulation. In that work (Titgemeyer et al., 2011), pharmacologic amounts of NA in cattle unexpectedly led to striking decreases in feed intake, which is a response different than that seen in nonruminants (Ramirez and Friedman, 1983; Real et al., 2002; Fukuwatari et al., 2009). Thus, the work described herein was initiated to determine the tissue distribution of GPR109A in cattle.

\section{MATERIALS AND METHODS}

\section{Tissue Distribution of GPR109A mRNA and Protein}

Experimental procedures were approved by the Kansas State University Institutional Animal Care and 
Use Committee. Six steers were used to evaluate the tissue distribution of GPR109A. The ruminally cannulated Holstein steers $(225 \pm 22 \mathrm{~kg})$ were managed in a trial to evaluate the effect of NA supply. Although the NA treatments did not affect GPR109A mRNA or protein (Table 1), the data are presented by treatment to demonstrate the robustness of the tissue distribution between treatments. In brief, cattle were managed as follows: steers were housed in metabolism crates and fed a grain-based diet ( $72 \%$ corn, $12 \%$ soybean meal, $10 \%$ alfalfa hay) containing $60 \mathrm{mg} / \mathrm{d}$ of zilpaterol (a $\beta$-adrenergic agonist that improves weight gain; Intervet Inc., Millsboro, DE) in amounts slightly less than ad libitum intake before the initiation of experiments. Infusion lines were placed through the ruminal cannulas and the omasum and into the abomasum, where they were anchored with a 10-cm-diameter rubber flange, to allow for continuous postruminal infusion of NA. Beginning $7 \mathrm{~d}$ before sample collection, NA treatments were initiated; 3 steers received continuous abomasal infusions of $16 \mathrm{~g} / \mathrm{d}$ of NA ( $\geq 99.5 \%$, Fluka Biochemika, Buchs, Switzerland) in $2 \mathrm{~L} / \mathrm{d}$ of water, and 3 steers received continuous abomasal infusions of water only. The initial plan was to maintain steers on their NA treatment for $7 \mathrm{~d}$, but the NA infusions were terminated after $3.2 \mathrm{~d}$ of treatment because cattle receiving $16 \mathrm{~g} / \mathrm{d}$ of NA demonstrated progressive reductions in voluntary feed intake (Titgemeyer et al., 2011). Steers were maintained on their diets through the initially planned trial (4 d after stopping NA treatment) and then euthanized with $187 \mathrm{mg}$ of sodium pentobarbital/ $\mathrm{kg}$ of BW followed by exsanguination.

After cattle were euthanized, tissue samples were collected from tail head fat, back fat, kidney fat, longissimus muscle, and liver. The collected tissues were immediately frozen in liquid nitrogen and later analyzed for mRNA abundance of bovine GPR109A and of the ribosomal protein subunit 9 (RPS9). Samples were analyzed for GPR109A protein by Western blot analysis.

\section{Sample Analysis}

Total RNA was isolated from tissues (Bonham and Danielpour, 1996) with a commercial kit (RNeasy Lipid Tissue Mini Kit, Qiagen Inc., Valencia, CA). Quality was verified with an Agilent 2100 Bioanalyzer (Agilent Technologies, Santa Clara, CA); RNA integrity number was $7.80 \pm 0.78$. Two micrograms of total RNA was used as template for the reverse transcriptase reaction using random primers (High-Capacity cDNA RT Kit, Applied Biosystems, Foster City, CA). Quantitative real-time reverse transcription (RT)-PCR was performed in triplicate with $1 / 20$ of the cDNA product in the presence of $200 \mathrm{nmol} / \mathrm{L}$ gene-specific forward and reverse primers with real-time SYBR green fluorescent detection using SYBR Green Premix reagent (7500 Fast Real-Time PCR System, Applied Biosystems). Primers were designed (www.ncbi.nlm.nih.gov) using GenBank accession no. XM_002701703.1 for GPR109A and no. DT860044.1 for RPS9. Regions amplified and primer sequences were as follows (all $5^{\prime}$ to $3^{\prime}$ ): GPR109A (901 to 1046); forward, ACATCACCCTCAGCTTCACC; reverse, GCGGTTGTTATCCGACTCAT; RPS9 (233 to 344); forward, GAACAAACGTGAGGTCTGGAGG; reverse, ATTACCTTCGAACAGACGCCG. Samples were run on 96-well plates, with all samples and standards for a gene located on 2 plates. For GPR109A mRNA expression, PCR performance criteria included $\mathrm{R}^{2}=0.9821$ and efficiency $=1.89$. The cycles to threshold $\left(\mathbf{C}_{\mathrm{t}}\right)$ for GPR109A were: $25.2 \pm 0.84$ for longissimus muscle, $22.4 \pm 0.41$ for kidney fat, $25.2 \pm 2.0$ for back fat, $23.9 \pm 3.3$ for tail head fat, and $22.3 \pm 1.7$ for liver. Relative mRNA abundance was quantified by the delta $\mathrm{C}_{\mathrm{t}}$ method with RPS9 to normalize values (JanovickGuretzky et al., 2007). The $\mathrm{C}_{\mathrm{t}}$ for RPS9 were not different among tissues $(P=0.28)$ nor were they affected by NA $(P=0.78)$ or NA by tissue interactions $(P=0.56)$.

Relative abundance of GPR109A protein in tissue samples was determined by Western blot. Tissue

Table 1. Abundance of GPR109A mRNA (relative to ribosomal protein subunit 9, RPS9) and GPR109A protein (densitometry units, $\times 10^{3}$ ) in steers supplemented with 0 or $16 \mathrm{~g} / \mathrm{d}$ of nicotinic acid $^{1}$

\begin{tabular}{lcc}
\hline & \multicolumn{2}{c}{ Nicotinic acid, g/d } \\
\cline { 2 - 3 } Item & 0 & 16 \\
\hline GPR109A mRNA $^{2}$ & & \\
Longissimus muscle $^{\mathrm{a}}$ & 0.062 & 0.064 \\
Kidney fat $^{\mathrm{bc}}$ & 0.146 & 0.155 \\
Back fat $^{\mathrm{ab}}$ & 0.117 & 0.108 \\
Tail head fat $^{\mathrm{c}}$ & 0.202 & 0.248 \\
Liver $^{\mathrm{d}}$ & 0.497 & 0.617 \\
GPR109A protein $^{3}$ & & \\
Longissimus muscle $^{\mathrm{ab}}$ & & \\
Kidney fat $^{\mathrm{c}}$ & 9.1 & 9.3 \\
Back fat $^{\mathrm{a}}$ & 13.6 & 19.0 \\
Tail head fat $^{\mathrm{c}}$ & 3.9 & 9.1 \\
Liver $^{\mathrm{bc}}$ & $16.6^{4}$ & 18.8 \\
\hline
\end{tabular}

${ }^{\mathrm{a}-\mathrm{d}}$ Within item, tissues without a common letter differ $(P<0.05)$.

${ }^{1}$ Steers received the nicotinic acid supplements for $3.2 \mathrm{~d}$, and the supplementation ended $4 \mathrm{~d}$ before tissue collection.

${ }^{2} \mathrm{SEM}=0.040$. Nicotinic acid, $P=0.31$; tissue, $P<0.0001$; nicotinic acid $\times$ tissue interaction, $P=0.48$.

${ }^{3} \mathrm{SEM}=3.0$. Nicotinic acid, $P=0.22$; tissue, $P=0.01$; nicotinic acid $\times$ tissue interaction, $P=0.86$. Three samples of back fat $(2$ for $0 \mathrm{~g} / \mathrm{d}$ nicotinic acid and 1 for $16 \mathrm{~g} / \mathrm{d}$ nicotinic acid) yielded no visible protein band. Densitometry analysis yielded values of $6.4 \times 10^{3}$ for the samples with no visible protein band, and this background value was subtracted from all samples for all tissues before statistical analysis.

${ }^{4}$ One missing sample for tail head fat for $0 \mathrm{~g} / \mathrm{d}$ nicotinic acid. 
samples $(\sim 20 \mathrm{mg})$ were homogenized at $4^{\circ} \mathrm{C}$ in RIPA lysis buffer (Santa Cruz Biotechnology, Santa Cruz, CA) containing a broad-spectrum protease inhibitor cocktail (Protease Inhibitor Cocktail I; Calbiochem, Gibbstown, NJ). The homogenate was centrifuged at $15,000 \times g$ for $10 \mathrm{~min}$ at $4^{\circ} \mathrm{C}$, and total protein concentration of the supernatant was measured (Bradford, 1976). Forty micrograms of total protein from tissues was diluted in Laemmli sample buffer, heated at $90^{\circ} \mathrm{C}$ for $5 \mathrm{~min}$, cooled, vortexed, separated by SDS-PAGE on a 4 to $12 \%$ Tris-HCl gel, and dry-transferred onto nitrocellulose membranes (iBlot; Invitrogen, Carlsbad, CA). Membranes were blocked in Tris buffer ( $\mathrm{pH} 7.4)$ with $5 \%$ dry milk powder for $2 \mathrm{~h}$ at room temperature and then incubated for $1 \mathrm{~h}$ at room temperature with a goat anti-HM74 antibody (1:1,000 dilution; Santa Cruz Biotechnology) raised against a short portion of the C-terminus of human GPR109A, which matched well with the predicted bovine sequence (the exact sequence of the epitope is proprietary). After washing, membranes were incubated up to $1 \mathrm{~h}$ at room temperature with secondary antibody (anti-goat IgG, Santa Cruz Biotechnology) diluted 10,000-fold in Tris buffer ( $\mathrm{pH}$ 7.4). Immunodetection was performed by chemiluminescence (West-Dura; Thermo Scientific, Waltham, MA), and bands were quantified by scanning densitometry (ChemiDoc-It Imaging System; UVP Inc., Upland, $\mathrm{CA})$.

\section{Brain Distribution of GPR109A mRNA}

Brain tissues were collected from 6 steers. Beef steers 12 to 16 mo of age were slaughtered at a local abattoir, and brain tissue was collected within 15 min postmortem from the cerebral cortex, cerebellum, thalamus, hypothalamus, and brain stem. For each tissue, samples were collected from the right and left sides of the brain and analyzed separately, and results from the 2 sides were averaged before statistical analysis. Collected samples were immediately placed in cold QIAzol lysis reagent (Qiagen Inc.) to prevent RNA degradation. Transcript abundance for GPR109A was determined relative to RPS9 for each of these regions of the brain as described above. The RNA integrity number for brain samples was $6.72 \pm 0.77$. The $\mathrm{C}_{\mathrm{t}}$ for GPR109A were $28.3 \pm 1.1$ in cerebral cortex, $27.8 \pm 1.2$ in cerebellum, $28.7 \pm 0.7$ in thalamus, $26.7 \pm 0.4$ in hypothalamus, and $28.4 \pm 1.4$ in brain stem.

\section{Gene Sequencing}

To verify the sequence of bovine GPR109A, the gene was sequenced using DNA isolated from 2 cattle. One animal was a steer used to evaluate tissue distribution of GPR109A, and the other was a Holstein cow not otherwise used for these trials. Genomic DNA was isolated from liver tissue (DNeasy Blood and Tissue Kit, Qiagen Inc.) and quantified by spectroscopy. Six primer sets were designed using bovine GenBank accession no. XM_002701703.1 to amplify overlapping regions of the gene. The PCR reaction was carried out as described for quantitative RT-PCR, except SYBR green was not included and a regular thermocycler was used. Amplicons were isolated (MinElute PCR Purification Kit, Qiagen Inc.), and the purity of the PCR products was verified by agarose gel separation with ethidium bromide detection. Amplicons were then sequenced by the Kansas State University Gene Sequencing Facility with a capillary sequencer (ABI 3730, Applied Biosystems). The assembled sequences were aligned against GenBank accession no. XM_002701703.1 using Clustal W (http://www.clustal.org).

\section{Immunofluorescence}

Samples of bovine (16-mo-old Holstein steer) liver were collected within $30 \mathrm{~min}$ after slaughter from a local abattoir, immediately embedded in Optimal Cutting Temperature Compound (Sakura Finetek, Torrance, CA), and frozen. Samples were sliced into $8-\mu \mathrm{m}$ sections and fixed in freshly prepared $4 \%$ formalin in PBS (pH 7.4) for 10 min. Sections were washed twice in PBS for $5 \mathrm{~min}$ and then drained, overlaid with 0.2 $\mathrm{mL}$ of primary goat anti-HM74 antibody (Santa Cruz Biotechnology) diluted 1:50 in PBS containing 0.2\% Triton X-100 (PBS-Tx) and 2\% BSA, and incubated overnight at $4^{\circ} \mathrm{C}$. Sections were washed 3 times with PBS-Tx and then overlaid with the secondary Alexa Fluor 488 anti-goat antibody (Invitrogen) diluted 1:1,000 for $45 \mathrm{~min}$ at room temperature in the dark. Sections were washed 3 times with PBS-Tx and stained with 4',6-diamidino-2-phenylindole (DAPI) and Alexa Fluor 594 phalloidin (Invitrogen). Sections were then observed with a confocal microscope equipped with laser scanning (LSM 510 Meta, Carl Zeiss, New York, NY).

\section{Statistical Analyses}

Abundances of GPR109A mRNA and protein were analyzed as a split-plot using the mixed procedure of SAS (SAS Inst. Inc., Cary, NC); the model included $\mathrm{NA}$, tissue, and NA $\times$ tissue interaction, and steer $\times$ NA interaction was a random effect that served as the main-plot error term. Abundance of GPR109A mRNA in brain samples was analyzed using a mixed model that included the fixed effect of sample location and the random effect of animal. Means were separated 
with pair-wise $t$-tests when the $F$-test demonstrated $P$ $<0.05$.

\section{RESULTS AND DISCUSSION}

\section{Tissue Distribution of GPR109A in Cattle}

The NA treatment did not affect abundance of GPR109A mRNA $(P=0.31)$ or protein $(P=0.22)$ in any of the tissues studied (Table 1). Although we report treatment means for all tissue samples to be comprehensive, the lack of a treatment response needs to be interpreted cautiously because the NA treatment was discontinued $4 \mathrm{~d}$ before tissues were collected, and the response to treatment was confounded by the effect of NA on feed intake. Expression of GPR109A could have been affected by the cattle's physiological responses to NA-mediated reductions in feed intake or to the subsequent increases in feed intake following termination of the NA treatment.

The mRNA for GPR109A was found in all tissues analyzed. Abundance of GPR109A mRNA was notably greater $(P<0.001)$ in liver than in the other tissues, which was surprising because it was absent from liver in other species (Tunaru et al., 2003; Wise et al., 2003). Recently, Li et al. (2010) demonstrated that basal expression of GPR109A was low in the liver of mice.

As expected, the kidney and tail head fat depots contained more $(P<0.05)$ GPR109A mRNA than the longissimus muscle, although back fat did not. The low concentrations of GPR109A mRNA in the longissimus muscle could reflect the presence of the GPR109A in bovine muscle tissue or the presence of adipocytes. Longissimus muscle of these steers did not appear to contain much fat, so it is probable that GPR109A was present on muscle cells.

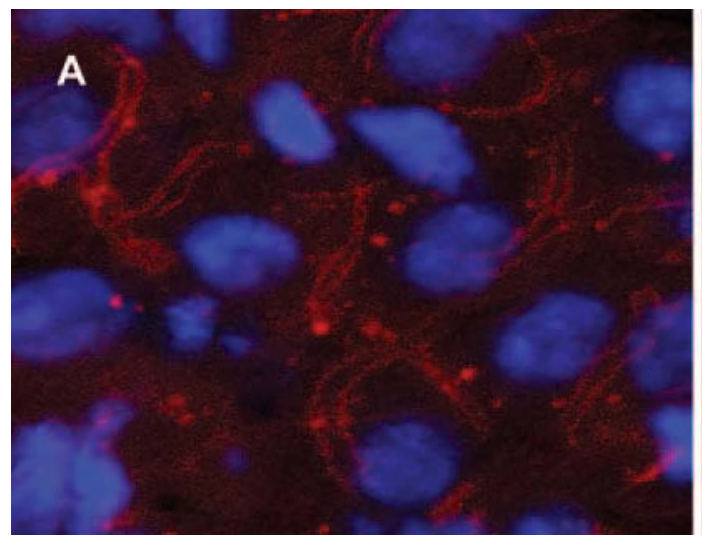

Because liver was not expected to contain large amounts of GPR109A, we were concerned that mRNA might be expressed without subsequent expression of the protein. Therefore, Western blot analysis was used to measure GPR109A proteins in the tissue samples. The GPR109A protein was observed in all samples of each tissue except for 3 back fat samples, for which no bands for GPR109A protein were observed (Table 1). No effect of NA treatment was observed for concentrations of GPR109A protein $(P=0.22)$. The band detected in the Western blot assay matched the expected molecular weight of bovine GPR109A, and no band appeared for mouse liver extract; both of these findings support the conclusion that the antibody detected the appropriate protein. This finding that cattle contain GPR109A in the liver is novel.

The comparatively large concentrations of GPR109A mRNA in the liver suggested that it was not isolated to a small population of immune cells but, at the same time, high expression of GPR109A mRNA was not expected in hepatocytes (Wise et al., 2003). Evaluation of liver tissue by confocal microscopy (Figure 1) suggests that GPR109A was expressed in parenchymal cells and not localized exclusively to immune-system cells. Similarly, Li et al. (2010) demonstrated that low level expression of GPR109A in mouse liver was specific to hepatocytes.

It is possible that GPR109A mediates the reductions in feed intake through receptors located in the brain, in addition to the liver. Miller and Dulay (2008) observed HM74A (GPR109A) expression in human brain, with lesser concentrations of the protein in brains of individuals with schizophrenia, suggesting a potential role of GRP109A in behavior regulation. The mRNA for GPR109A was detected in all regions of bovine brain analyzed (Figure 2). Although GPR109A transcript

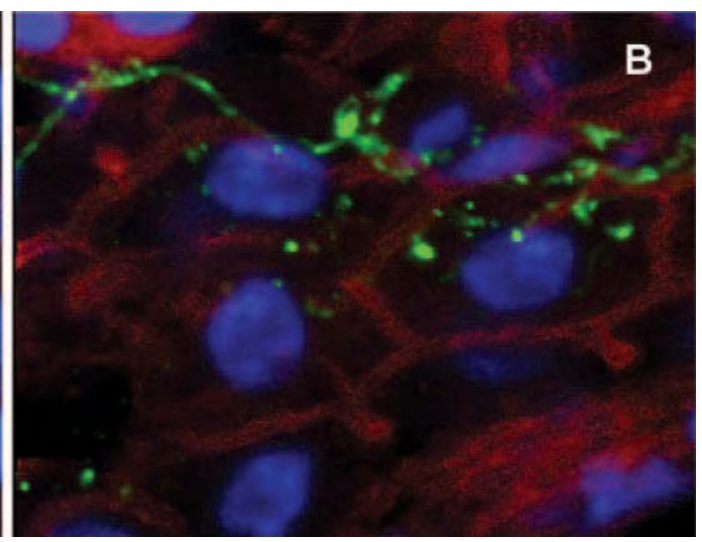

Figure 1. Localization of GPR109A (niacin receptor) in bovine liver by confocal microscopy. Sections of bovine liver were stained to detect DNA [4',6-diamidino-2-phenylindole (DAPI): blue/circular shapes] and filamentous actin (phalloidin: red/hexagonal shapes). Samples incubated without (A) and with (B) primary anti-GPR109A antibody were incubated with a green fluorescent-labeled secondary antibody (brighter spots in panel B). Magnification: $40 \times$. Color version available in the online PDF. 


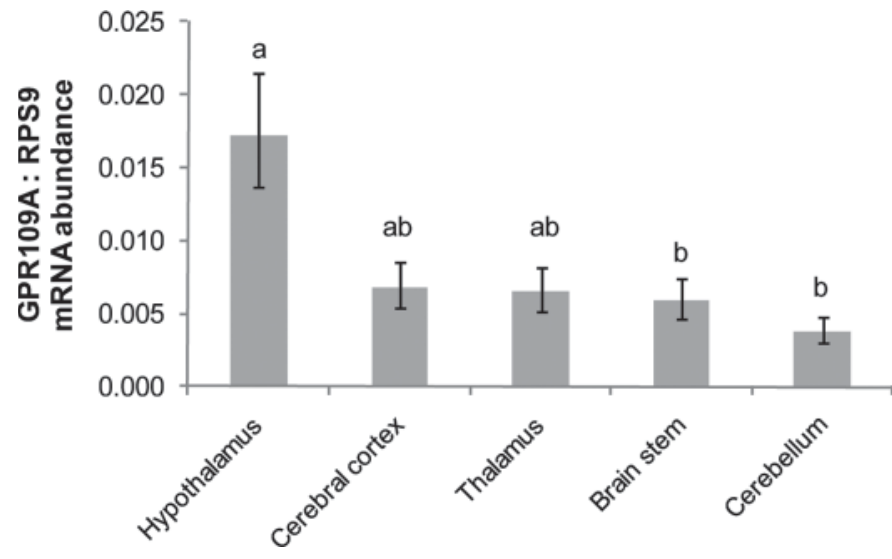

Figure 2. Detection of GPR109A (niacin receptor) mRNA in bovine brain tissue. Samples of indicated brain structures were collected from steers at slaughter, and mRNA abundance was determined by quantitative real-time reverse-transcription PCR. Values are means \pm SEM, $\mathrm{n}=6$. Means without a common letter differ, $P<0.05$.

abundance was relatively low in the brain compared with other tissues analyzed (Table 1), it was most abundant in the hypothalamus, the region of the brain that ultimately controls feeding behavior (Sartin et al., 2011)

\section{Identification of the Bovine Niacin Receptor Sequence}

The human genome includes 2 highly homologous receptors that bind to niacin, GPR109A and GPR109B. Although these proteins share $96 \%$ sequence homology, GPR109A binds NA and BHBA with high affinity, whereas GPR109B has far lower affinity for these ligands (Gille et al., 2008). By searching the bovine genome for homologs to these 2 sequences, we retrieved a single sequence (GenBank accession no. XM_002701703.1) with 83 and $81 \%$ AA homology to human GPR109A and GPR109B, respectively. Gene sequencing from 2 cattle verified the accuracy of the XM_002701703.1 sequence, revealing only a silent $\mathrm{T} / \mathrm{C}$ mutation at nucleotide 814 . The identification of a single sequence in the bovine genome is consistent with evidence that the presence of 2 niacin receptors in the human genome is the result of a recent gene duplication event (Wise et al., 2003). Sitedirected mutagenesis revealed several AA in GPR109A that confer its increased affinity for NA compared with GPR109B (Tunaru et al., 2005); this allowed assessment of the likelihood that the single bovine sequence encodes a protein that is responsive to NA. Each AA residue in GPR109A that was determined critical for NA binding (Tunaru et al., 2005) matched with the bo-

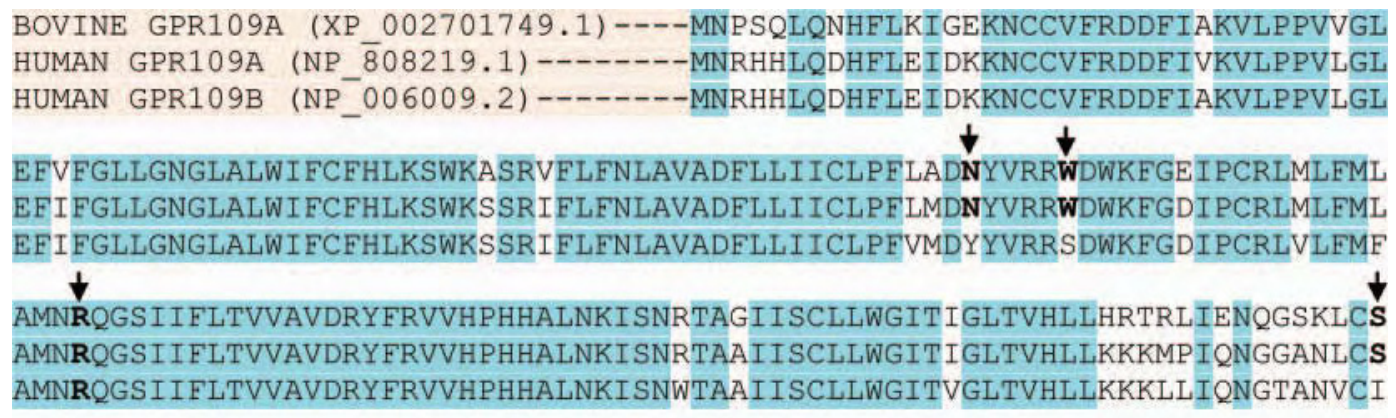

SFS ICDAFRWHDAMFLLEF FVPLGI ILFCSVRIVWSLRQRQMNKHAKIRRAINFIMVVAIVF I ICFLPSVA SES ICHTFQWHEAMFLLEFFLPLGI I LFCSAR I IWSLRQRQMDRHAKIKRA ITF IMVVAIVFVICFLPSVV SES ICHTFRWHEAMFLLEFFLPLGI ILFCSARI IWSLRQRQMDRHAKIKRAITFIMVVAIVFVICFLPSVV VRIHIFWLLRKAGTENCDIYRSVDLAFYITLSETYMNSMLDPLVYYFSSPSEPNEFSTLINRCLKRKGPDE VRIRI FWLLHTSGTQNCEVYRSVDLAFFITLSFT YMNSMLDPVVYYFSSPSFPNFFSTLINRCLQRKMTGE VRIHIFWLLHTSGTQNCEVYRSVDLAFFITLSFTYMNSMLDPVVYYFSSPSFPNFFSTLINRCLQRKITGE

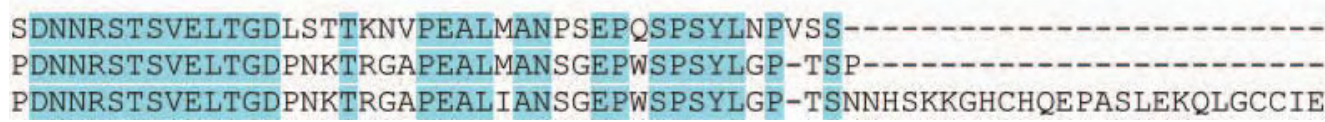

Figure 3. Identification of bovine GPR109A (niacin receptor). Bovine sequence XP_002701749.1 (the protein sequence corresponding to XM_002701703.1) was aligned against human GPR109A and GPR109B. Regions that are uniform across all 3 proteins are shaded (highlighted). The bovine sequence shared 83\% homology with human GPR109A and 81\% homology with human GPR109B. Residues in human GPR109A indicated by arrows are critical for binding niacin (Tunaru et al., 2005); the bovine sequence matches GPR109A at each of these 6 positions, whereas human GPR109B differs at 3 of them. The predicted molecular weight of bovine GPR109A is 41.6 kDa. Color version available in the online PDF. 
vine sequence encoded by XM_002701703.1 (Figure 3). This sequence similarity between XM_002701703.1 and GPR109A, along with the antilipolytic effect of pharmacological doses of NA in cattle (Pires and Grummer, 2007; Titgemeyer et al., 2011), suggests that bovine sequence XM_002701703.1 encodes a functional niacin receptor.

The GPR109A receptor may not act in cattle as it does in other species (in terms of agonists that stimulate it and effects that it wields). The location of GPR109A in the bovine body leads to many interesting questions related to feed intake regulation and metabolism in cattle. The ability of BHBA to bind and activate GPR109A (Gille et al., 2008), coupled with the prevalence of ketosis in dairy cattle (Krebs, 1966), makes the unusual distribution of GPR109A in cattle especially intriguing. Recent research demonstrated the ability of the hypothalamus to sense and respond to fatty acids, glucose, and AA (Moran, 2010). It is conceivable that, at least in ruminants, GPR109A allows the central nervous system to monitor circulating BHBA concentrations, and that intake depression by NA is mediated by GPR109A in the brain.

\section{CONCLUSIONS}

High concentrations of GPR109A mRNA were observed in the liver, and Western blot analysis showed the presence of GPR109A protein in liver tissue. It is possible that NA acts directly on brain because GPR109A (at least mRNA) was observed in bovine brain.

\section{ACKNOWLEDGMENTS}

This article is contribution no. 10-350-J from the Kansas Agricultural Experiment Station, Manhattan. Partial financial support was provided by Lonza (Allendale, NJ). We thank the COBRE Confocal Microfluorometry and Microscopy Core at Kansas State University, funded by National Institutes of Health Grant P20 RR-017686, for generous support. We are grateful for the technical assistance in confocal microscopy provided by Joel Sanneman at Kansas State University (Manhattan).

\section{REFERENCES}

Bonham, M. J., and D. Danielpour. 1996. Improved purification and yields of RNA by RNeasy. Biotechniques 21:57-60.

Bradford, M. M. 1976. A rapid and sensitive method for the quantitation of microgram quantities of protein utilizing the principle of protein-dye binding. Anal. Biochem. 72:248-254.

Fukuwatari, T., K. Kurata, and K. Shibata. 2009. Effects of excess nicotinic acid on growth and the urinary excretion of B-group vita- mins and the metabolism of tryptophan in weaning rats. Shokuhin Eiseigaku Zasshi 50:80-84.

Gille, A., E. T. Bodor, K. Ahmed, and S. Offermanns. 2008. Nicotinic acid: Pharmacological effects and mechanisms of action. Annu. Rev. Pharmacol. Toxicol. 48:79-106.

Janovick-Guretzky, N. A., H. M. Dann, D. B. Carlson, M. R. Murphy, J. J. Loor, and J. K. Drackley. 2007. Housekeeping gene expression in bovine liver is affected by physiological state, feed intake, and dietary treatment. J. Dairy Sci. 90:2246-2252.

Krebs, H. A. 1966. Bovine ketosis. Vet. Rec. 78:187-192.

Lee, D. K., T. Nguyen, K. R. Lynch, R. Cheng, W. B. Vanti, O. Arkhitko, T. Lewis, J. F. Evans, S. R. George, and B. F. O'Dowd. 2001. Discovery and mapping of ten novel G protein-coupled receptor genes. Gene 275:83-91.

Li, X., J. S. Millar, N. Brownell, F. Briand, and D. J. Rader. 2010. Modulation of HDL metabolism by the niacin receptor GPR109A in mouse hepatocytes. Biochem. Pharmacol. 80:1450-1457.

Martin, P. M., S. Ananth, G. Cresci, P. Roon, S. Smith, and V. Ganapathy. 2009. Expression and localization of GPR109A (PUMA$\mathrm{G} / \mathrm{HM} 74 \mathrm{~A})$ mRNA and protein in mammalian retinal pigment epithelium. Mol. Vis. 15:362-372.

Miller, C. L., and J. R. Dulay. 2008. The high-affinity niacin receptor HM74A is decreased in the anterior cingulate cortex of individuals with schizophrenia. Brain Res. Bull. 77:33-41.

Moran, T. H. 2010. Hypothalamic nutrient sensing and energy balance. Forum Nutr. 63:94-101.

Pires, J. A. A., and R. R. Grummer. 2007. The use of nicotinic acid to induce sustained low plasma nonesterified fatty acids in feedrestricted Holstein cows. J. Dairy Sci. 90:3725-3732.

Pires, J. A. A., J. B. Pescara, and R. R. Grummer. 2007. Reduction of plasma NEFA concentration by nicotinic acid enhances the response to insulin in feed-restricted Holstein cows. J. Dairy Sci. 90:4635-4642.

Ramirez, I., and M. I. Friedman. 1983. Metabolic concomitants of hypophagia during recovery from insulin-induced obesity in rats. Am. J. Physiol. 245:E211-E219.

Real, D. E., J. L. Nelssen, J. A. Unruh, M. D. Tokach, R. D. Goodband, S. S. Dritz, J. M. DeRouchey, and E. Alonso. 2002. Effects of increasing dietary niacin on growth performance and meat quality in finishing pigs reared in two different environments. J. Anim. Sci. 80:3203-3210.

Sartin, J. L., B. K. Whitlock, and J. A. Daniel. 2011. Neural regulation of feed intake: Modification by hormones, fasting and disease. J. Anim. Sci. 89:1991-2003. doi:10.2527/jas.2010-3399.

Thangaraju, M., G. A. Cresci, K. Liu, S. Ananth, J. P. Gnanaprakasam, D. D. Browning, J. D. Mellinger, S. B. Smith, G. J. Digby, N. A. Lambert, P. D. Prasad, and V. Ganapathy. 2009. GPR109A is a G-protein-coupled receptor for the bacterial fermentation product butyrate and functions as a tumor suppressor in colon. Cancer Res. 69:2826-2832.

Titgemeyer, E. C., K. S. Spivey, L. K. Mamedova, and B. J. Bradford. 2011. Effects of pharmacological amounts of nicotinic acid on lipolysis and feed intake in cattle. Int. J. Dairy Sci. 6:134-141.

Tunaru, S., J. Kero, A. Schaub, C. Wufka, A. Blaukat, K. Pfeffer, and S. Offermanns. 2003. PUMA-G and HM74 are receptors for nicotinic acid and mediate its anti-lipolytic effect. Nat. Med. 9:352-355

Tunaru, S., J. Lattig, J. Kero, G. Krause, and S. Offermanns. 2005. Characterization of determinants of ligand binding to the nicotinic acid receptor GPR109A (HM74A/PUMA-G). Mol. Pharmacol. 68:1271-1280.

Wise, A., S. M. Foord, N. J. Fraser, A. A. Barnes, N. Elshourbagy, M. Eilert, D. M. Ignar, P. R. Murdock, K. Steplewski, A. Green, A. J. Brown, S. J. Dowell, P. G. Szekeres, D. G. Hassall, F. H. Marshall, S. Wilson, and N. B. Pike. 2003. Molecular identification of high and low affinity receptors for nicotinic acid. J. Biol. Chem. 278:9869-9874. 